\title{
Study on the economic disparity and convergence of urban agglomeration in the middle reaches of the Yangtze River
}

\author{
Chao Wen, Jingjing Chen \\ Department of Finance and Economics, Guangdong University of Science and Technology, Dongguan, China
}

Keywords: The Middle Reaches Of The Yangtze River City Group; Economic Gap; Convergence; Exploratory Spatial Data Analysis; Spatial Error Model.

\begin{abstract}
The economic gap is an important indicator of a regional economic development. This paper is about 31 cities in the middle reaches of the Yangtze River City Group years from 2003 to 2013, a total of 11 years of data as the basis, using spatial difference index to analysis the gap between the economics of developing cities. While introduction of a spatial error model and a spatial lag model of the spatial regression analysis to provide theoretical support for the convergence of its economic development. Comprehensive measure the economic development coordination of the middle reaches of the Yangtze River City Group in the past 10 years to development the regions better in the future.
\end{abstract}

\section{Introduction}

So far, there is no specialized research for the disparity and convergence of the middle reaches of the Yangtze River city group. This paper will analysis the gap between the level of regional economic development, integrated by spatial difference analysis, such as Coefficient of Variation, Theil Entropy Index, Hirfindhal-Hirschman index and Gini coefficient. Then introduce a spatial error model and a spatial lag model of the spatial regression analysis to provide a theoretical support for the convergence of the regional economic development. Measure the balance of the economic development in the middle reaches of the Yangtze River City Group in the past 10 years, for the future development of the region.

\section{Method}

Kinds of spatial indexes utilize on the study of the economic disparity. There are many indexes in measuring regional economic development gap. The most commonly used are Coefficient of Variation (CV), Theil Entropy Index (TEI), Hirfindhal-Hirschman Index (HHI) and Gini coefficient (Gini). In order to comprehensively measure the economic development gap between the Yangtze River city group, the economic gap will be integrated with the four indicators all above to measure the area, in order to reflect the real situation in the region.

Comparison of spatial error model and a spatial lag model of regional to measure the spatial correlation and convergence characteristics. Introduces the spatial econometrics model to accurately assess the middle reaches of the Yangtze River city group economic gap is convergence or not in the past ten years. Starting from the analysis of the regional spatial autocorrelation, then compare the use of spatial error model (SEM) and spatial lag model (SLM) to analyze the convergence characteristics of the regional economy.

General Measurement Model (GMM). The form of the GMM is as follows:

$$
\ln \left(\frac{y_{i T}}{y_{i O}}\right)=\alpha+\beta \ln \left(y_{i O}\right)+\varepsilon_{i}
$$


In the model I, $y_{i T}$ for the city of i final per capita national income level, $y_{i O}$ for the city of $\mathrm{i}$ beginning per capita income level. This model reflects the relationship between the independent variable $y_{i O}$ and the dependent variable $\frac{y_{i T}}{y_{i O}}$, if the coefficient $\beta$ is positive, the divergence of regional economic development is positive, and the negative is convergent.

Spatial Error Model (SEM). The spatial error model is constructed as follows:

$$
\left\{\begin{array}{l}
\ln \left(\frac{y_{i T}}{y_{i O}}\right)=\alpha+\rho W_{y}+\beta \ln \left(y_{i O}\right)+\varepsilon_{i} \\
\varepsilon_{i}=\lambda W_{\varepsilon}+\mu
\end{array}\right.
$$

In the model II, $\lambda$ is the error term of the spatial autoregressive coefficient, $W_{\varepsilon}$ is spatial error spatial lag vector, $\mu$ is not related, mean value is 0 , with the same variance of the error term. This model is added spatial weight matrix and the spatial error effect, its positive and negative coefficient $\beta$ also reflects the gap between the regional economic divergence or convergence.

Spatial Lag Model (SLM). Construction of the Spatial Lag Model is as follows:

$$
\ln \left(\frac{y_{i T}}{y_{i O}}\right)=\alpha+\rho W_{y}+\beta \ln \left(y_{i O}\right)+\varepsilon_{i}
$$

In the model III, ${ }^{W_{y}}$ is due to the variable spatial lag vectors, which is an endogenous variable, the coefficient $\rho$ reflects the effect of neighbors on the region itself, the other coefficient same model I and II.

\section{Results}

General development level and gap of the region. Compared with the mainstream analysis methods at home and abroad, we learn from Wei Houkai (1997) using the per capita GDP as an indicator to measure the convergence of China's regional economy. To collect the data of per capita GDP of the urban agglomeration in the middle reaches of the Yangtze River, 2003-2013, the middle reaches of the Yangtze River City Group in 2003 and 2013 differences in per capita GDP spatial structure diagram, as follows:
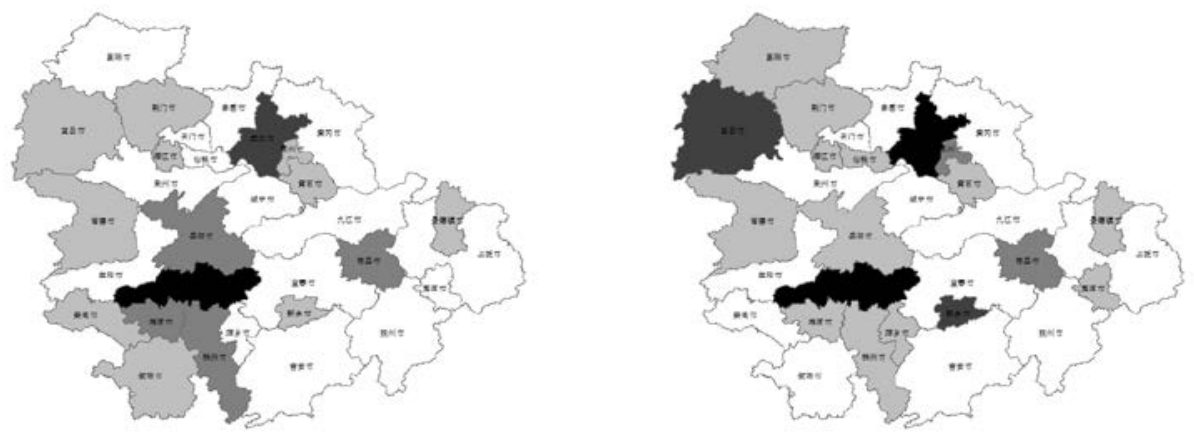

PCGDP spatial difference chart in 2003 PCGDP spatial difference chart in 2013

Figure 1: Comparison the per capita GDP spatial differences of urban agglomeration in the middle reaches of the Yangtze River in 2003 and 2013

According to the above is not difficult to find, compared to 2003, in 2013 the Yangtze River middle reaches of urban agglomeration economic spatial development more balanced. Such as Changsha, the radiation effect is more obvious, the surrounding cities get better development, formation of Chang-zhu-tan urban agglomeration. Another example is Hubei Province, in addition to Wuhan, grow out of Yichang as the second development poles to promote the development of the surrounding cities. In Jiangxi Province, such as Nanchang, Xinyu, Yingtan, Jingdezhen and other 
cities have started the signs of development, the future will form a new growth pole, multiple points to promote the common development of the surrounding cities. All of the above shows that the overall economic development gap narrowing in the past 10 years, cultivate a number of new growth poles to promote the common development of the surrounding cities, showing signs of convergence of economic development of the middle reaches of the Yangtze River city group.

Measure the development trend of regional economic disparity. In order to determine the gap of regional economic development more accurately, we use the R language calculated the indexes of economic development level in the middle reaches of the Yangtze River city group, the result of the Coefficient of Variation (CV), Theil Entropy Index (TEI), Hirfindhal-Hirschman Index (HHI) and Gini coefficient (Gini) showed in the following table:

Table 1 The indexes of per capita GDP 2003-2013

\begin{tabular}{ccccc}
\hline & Gini & Theil & var & hhi \\
\hline $\mathbf{2 0 0 3}$ & 0.2655 & 0.1160 & 0.5157 & 0.0408 \\
$\mathbf{2 0 0 5}$ & 0.2388 & 0.0928 & 0.4528 & 0.0388 \\
$\mathbf{2 0 0 7}$ & 0.2420 & 0.0936 & 0.4518 & 0.0388 \\
$\mathbf{2 0 0 9}$ & 0.2536 & 0.1060 & 0.4893 & 0.0399 \\
$\mathbf{2 0 1 1}$ & 0.2463 & 0.0973 & 0.4604 & 0.0390 \\
$\mathbf{2 0 1 3}$ & 0.2395 & 0.0914 & 0.4452 & 0.0386 \\
\hline
\end{tabular}

From the calculation results of the Gini coefficient, Theil Index, Coefficient of Variation and Hirfindhal-Hirschman Index, the economic development of the urban agglomeration in the middle reaches of the Yangtze River shows a certain degree of difference in each year. They have changed over the years, but the overall evolution has a downward trend. From the view of the trend of the change of the four, its trajectory is also quite consistent in the undulating stage. Presents the first increased and then decreased the inverted "U" - type movements, the inflection point occurred in 2009, especially the Theil index is particularly evident. In this note, the economic gap of the middle reaches of the Yangtze River city group before 2009 is a divergent trend. In recent years, the trend is convergent, suggesting that regional economic coordination is getting better and better in recent years, as it shown in figure 2:

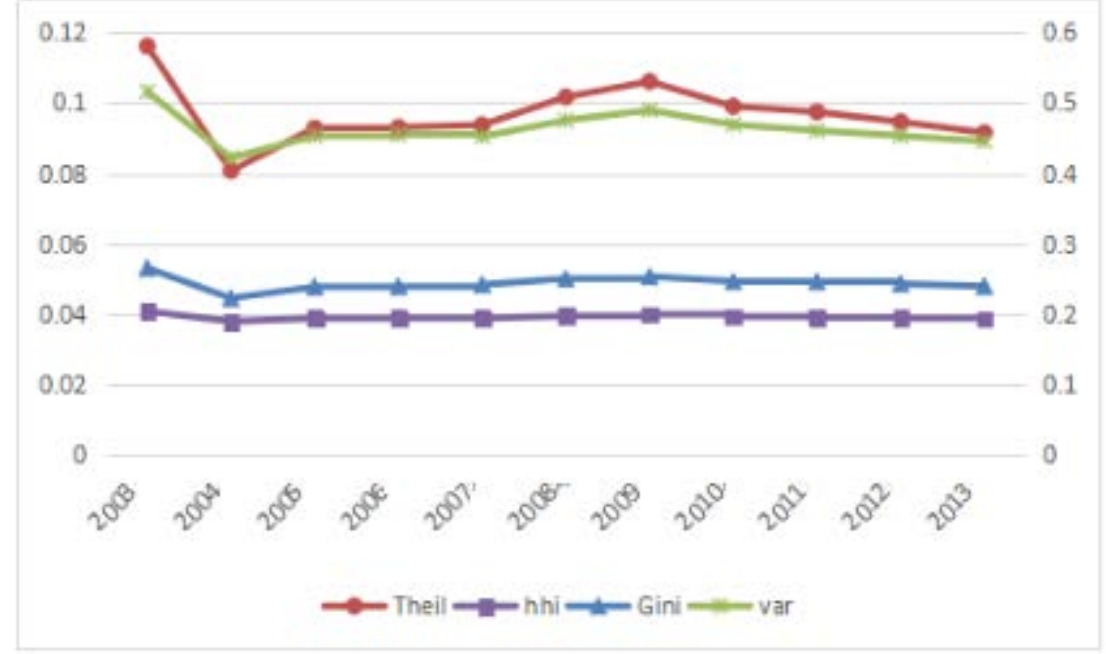

Figure 2. Trajectory of four kinds of gap indexes 


\section{Discussion}

General measurement model and test. In order to further measure the convergence of the economic development level of the urban agglomeration in the middle reaches of the Yangtze River, we introduce an general measurement model I. According to the model above, the results of the analysis using $\mathrm{R}$ software are as follows:

Table 2 The general measurement model estimation results

\begin{tabular}{ccccc}
\hline & Estimate & Std.Error & $\mathrm{t}$ value & $\operatorname{Pr}(>|\mathrm{t}|)$ \\
\hline$\alpha$ & 3.6478 & 0.8301 & 4.395 & 0.0001 \\
$\beta$ & -0.2370 & 0.0911 & -2.602 & 0.0144 \\
standard error & 0.2347 & & & \\
$\mathrm{R}^{2}$ & 0.1893 & & & \\
Adjusted R $^{2}$ & 0.1613 & & & \\
F-statistic & 6.77 & & & 0.0145 \\
\hline
\end{tabular}

Results from the convergence analysis can be found that the regression coefficient is -0.2370 , the $p$ value is 0.0144 , which is significant at the 0.05 level. This indicates that the economic development speed of the urban agglomeration in the middle reaches of the Yangtze River is negatively related to the beginning of the period. In fact, judging from the various indicators of regional economic development gap, their average trend in the year of the survey is also declining, as shown in figure 2 . At the same time, we found that the development of the urban agglomeration in the middle reaches of the Yangtze River may be related to the spatial correlation, the calculation model of the Moran's I. It can be found that Moran's I $=0.3683, \mathrm{P}=0.0002$, is very significant, which indicates that there is a certain spatial autocorrelation in the economic development of the urban agglomeration in the middle reaches of the Yangtze river. Taking into account the possible existence of spatial lag or spatial error in the model, continuing to analyze with the spatial error model as follows.

4.2 Spatial error model analysis and verification

The construction and significance of spatial error model are found in the model II above. According to the above model, the results of the analysis using $\mathrm{R}$ software are as follows:

Table 3 Spatial error model estimation results

\begin{tabular}{ccccc}
\hline & Estimate & Std.Error & $\mathrm{z}$ value & $\operatorname{Pr}(>|\mathrm{z}|)$ \\
\hline$\alpha$ & 2.5536 & 0.6827 & 3.7406 & 0.0002 \\
$\beta$ & -0.1129 & 0.0738 & -1.529 & 0.1263 \\
$\lambda$ & 0.5940 & & & 0.0329 \\
std.error & 3.626 & & & 0.0003 \\
Wald statistic & 13.148 & & & 0.0003 \\
Log likelihood & 4.2538 & & & \\
AIC & -0.5075 & & & \\
\hline
\end{tabular}

It can be found that compared with the results of general econometric model to the explained variable of the spatial error model return coefficient also is negative, and the interpretation of the results is the same. Spatial autoregressive coefficient $\lambda=0.5940$, $\mathrm{P}$ value $=0.0003$, significant. At the same time, the Akaike Information Criterion (AIC) of the spatial error model is - 0.5075 , which is less than the AIC value of the OLS estimation model 2.044. After adding the spatial error, the model can be improved, the only problem is that the $\mathrm{P}$ value of the model is not very significant.

Spatial lag model analysis and verification. Try to improve the model I with the spatial lag model III, and see if we can get a better result. According to the above model III, the results of the analysis using $\mathrm{R}$ software are as follows: 
Table 4 Spatial lag model estimation results

\begin{tabular}{ccccc}
\hline & Estimate & Std.Error & $\mathrm{z}$ value & $\operatorname{Pr}(>|\mathrm{z}|)$ \\
\hline$\alpha$ & 2.2204 & 0.7533 & 2.9475 & 0.0032 \\
$\beta$ & -0.1720 & 0.0757 & -2.2708 & 0.0232 \\
$\rho$ & 0.5679 & & & 0.0081 \\
A std.error & 0.1586 & & & 0.0003 \\
Wald statistic & 12.818 & & & 0.0003 \\
Log likelihood & 5.4860 & & & \\
AIC & -2.972 & & & \\
test value & 0.3276 & & & 0.5671 \\
\hline
\end{tabular}

It can be found that the regression coefficients of the model explanatory variables are equally significant. The regression coefficient of $\log (\mathrm{Y} 2003)$ was -0.1720 , and the $\mathrm{p}$ value was 0.0232 , which was significant. Spatial autoregressive coefficient $\rho=0.5679$, P-value $=0.0003$, are very significant too, which indicates that there is a strong spatial auto regression in the urban agglomeration in the middle reaches of the Yangtze River. At the same time, the Akaike Information Criterion (AIC) of the spatial error model is -2.972, which is less than the AIC value of the OLS estimation model 2.044. After adding the space lag variable, the model can be improved effectively.

\section{Conclusion}

Through the spatial exploration of graphic analysis. After the emergence of the development pole drive, the diffusion effect that the surrounding common development is obvious, such as Chang-zhu-tan city group. As well as, foster the development of a new growth pole to drive growth developments, such as the rise of Yichang.

In the comprehensive survey of the regional economic growth, and fond the area have the obvious spatial correlation. The convergence of economic development of the urban agglomeration in the middle reaches of the Yangtze River is explained.

To compare measure spatial lag model and spatial error model. The regression coefficient shows that the economic growth rate is negatively correlated with the total economic output in the initial years, that is, the regional economic development convergence. In fact, from spatial exploration of graphic analysis and regional economic disparities of various indicators, have confirmed the convergence characteristics of the regional economic development in the middle reaches of the Yangtze River City Group in the past 10 years..

\section{References}

[1] Qi Shaozhou,Yun Bo,Li Kai. An International Comparative Analysis on China's Economic Growth and the Convergence in Energy Intensity Gap and Its Economic Mechanism[J]. Chinese Journal of Population,Resources and Environment,2011,02:65-75.

[1] Guo Jiqiang. Measurement and decomposition methods of income gap (English). Social Sciences in China [J]., 2011,03:181-202.

[3] Xie Hualin, Wang Wei. China's major economic city industrial zone with spatial and temporal differences in efficiency and convergence analysis (English) [J]. Journal of Geographical Sciences, 2015,10:1183-1198. 